\title{
Astrophysics
}

\section{NGC 4254: a spiral galaxy entering the Virgo cluster ${ }^{\star}$}

\author{
B. Vollmer ${ }^{1,2}$, W. Huchtmeier ${ }^{2}$, and W. van Driel ${ }^{3}$ \\ ${ }^{1}$ CDS, Observatoire astronomique de Strasbourg, UMR 7550, 11 rue de l'Université, 67000 Strasbourg, France \\ e-mail: bvollmer@astro.u-strasbg.fr \\ 2 Max-Planck-Institut für Radioastronomie, Auf dem Hügel 69, 53121 Bonn, Germany \\ 3 Observatoire de Paris, Section de Meudon, GEPI, CNRS UMR 8111 et Université de Paris 7, 5 place Jules Janssen, \\ 92195 Meudon Cedex, France
}

Received 25 May 2004 / Accepted 29 March 2005

\begin{abstract}
Deep Effelsberg HI spectra of the one-armed, bright Virgo cluster spiral galaxy NGC 4254 are presented. Five different positions were observed in the $21 \mathrm{~cm} \mathrm{HI}$ line with the Effelsberg 100-m telescope: one in the center and 4 located one HPBW to the NE, NW, SW, and SE, respectively, from the galaxy center. The spectra are compared to existing deep VLA observations, and the single dish and interferometric HI data are used to constrain a dynamical model which includes the effects of ram pressure. The peculiar, one-armed spiral pattern of NGC 4254 and its distorted and kinematically perturbed atomic gas distribution can be explained by a close and rapid encounter $\sim 280 \mathrm{Myr}$ ago with another massive Virgo galaxy, followed by ram pressure stripping that is ongoing. The stripping occurs almost face-on, since the angle between the disk and the orbital plane is $70^{\circ}$. The galaxy with which NGC 4254 had its encounter is tentatively identified as the lenticular NGC 4262.
\end{abstract}

Key words. galaxies: individual: NGC 4254 - galaxies: interactions - galaxies: ISM - galaxies: kinematics and dynamics

\section{Introduction}

NGC 4254 is a bright spiral galaxy located at the periphery of the Virgo cluster, at a projected distance of $3.7^{\circ}\left(\sim 1 \mathrm{Mpc}^{1}\right)$ from the cluster center (M 87). Its basic parameters are listed in Table 1. The spiral structure of NGC 4254 shows an important $m=1$ mode, giving it a one-armed appearance (Fig. 5). The $m=2$ mode, which is prominent in most grand-design spirals, is almost absent. Iye et al. (1982) suggested that the intrinsic properties of NGC 4254 are responsible for the predominant $m=1$ mode.

Phookun et al. (1993) obtained deep HI $21 \mathrm{~cm}$ line observations of this galaxy with the VLA. These deep observations revealed, in addition to the galactic HI disk component, HI clouds superposed on and beyond the HI disk with velocities that do not follow the disk rotation pattern. They also found very low surface density atomic gas up to $\sim 6^{\prime}(\sim 30 \mathrm{kpc})$ to the north-west of the galaxy center. Based on their observations, Phookun et al. (1993) suggested that the low surface density gas represents a remnant of an entity that was tidally disrupted by NGC 4254 and is now merging with it. In this scenario the $m=1$ spiral structure was stimulated by the infalling gas.

In many respects NGC 4254 resembles NGC 4654 (Phookun \& Mundy 1995), another bright Virgo cluster spiral galaxy that is located at about the same projected distance from the cluster center but at the opposite side of the cluster,

$\star$ Movies are only available in electronic form at http://www . edpsciences.org

1 We use $D=17 \mathrm{Mpc}$ for the distance to the Virgo cluster.
Table 1. Physical parameters of NGC 4254.

\begin{tabular}{|c|c|}
\hline \multirow[t]{3}{*}{ Other names } & M 99 \\
\hline & VCC 307 \\
\hline & CGCG $098-144$ \\
\hline$\alpha(2000)^{a}$ & $12^{\mathrm{h}} 18^{\mathrm{m}} 49.6^{\mathrm{s}}$ \\
\hline$\delta(2000)^{a}$ & $14^{\circ} 24^{\prime} 59^{\prime \prime}$ \\
\hline Morphological type ${ }^{a}$ & $\mathrm{Sc}$ \\
\hline Distance to the cluster center $\left({ }^{\circ}\right)$ & 3.7 \\
\hline Optical diameter $D_{25}{ }^{a}\left(^{\prime}\right)$ & 5.4 \\
\hline$B_{\mathrm{T}}^{0 a}$ & 10.44 \\
\hline Systemic heliocentric velocity ${ }^{a}\left(\mathrm{~km} \mathrm{~s}^{-1}\right)$ & $2407 \pm 3$ \\
\hline Distance $D(\mathrm{Mpc})$ & 17 \\
\hline$V \operatorname{rot}_{\max }\left(\mathrm{km} \mathrm{s}^{-1}\right)$ & $150^{b}$ \\
\hline Major axis pa & $68^{\circ} b$ \\
\hline Inclination angle & $42^{\circ b}$ \\
\hline HI deficiency ${ }^{c}$ & $0.17 \pm 0.2$ \\
\hline
\end{tabular}

${ }^{a}$ RC3, de Vaucouleurs et al. (1991).

${ }^{b}$ Phookun et al. (1993).

${ }^{c}$ Cayatte et al. (1994).

since both galaxies (i) show a prominent $m=1$ spiral structure; (ii) are not HI deficient; and (iii) have an extended, very low surface density gas tail.

Vollmer (2003) showed that the $m=1$ spiral structure of NGC 4654 can be explained by a tidal interaction of NGC 4654 with its companion NGC 4639 , which took place $\sim 500 \mathrm{Myr}$ 
ago, while its extended gas tail can be explained by ongoing ram pressure stripping (Gunn \& Gott 1972). Since the outer part of the gas disk was perturbed by the gravitational interaction, only a small amount of ram pressure is needed to create the gas tail. Thus, only the combination of a close encounter with NGC 4639 followed by continuing moderate ram pressure can reproduce all observed properties of NGC 4654. In this article we investigate if the same evolutionary scenario involving a close encounter and ongoing ram pressure stripping applies to NGC 4254.

The Effelsberg observations are described in Sect. 2, followed by the results in Sect. 3. The model is introduced in Sect. 4. We present two different simulations in Sect. 5: (i) a close and rapid encounter between NGC 4254 and another massive Virgo cluster galaxy (Sect. 5.1) and (ii) a close and rapid encounter together with ram pressure stripping (Sect. 5.2). The simulations are compared to HI observations in Sect. 5.3 and discussed in Sect. 6, followed by our conclusions in Sect. 7.

\section{Observations}

Between January and March 2002 we performed 21-cm line observations with the $100 \mathrm{~m}$ Effelsberg telescope at 5 different positions in and around NGC 4254 at a frequency corresponding to the systemic velocity of NGC 4254 and a bandwidth of $12.5 \mathrm{MHz}$. The two-channel receiver had a system noise of $\sim 30 \mathrm{~K}$. The 1024 channel autocorrelator was split into four banks of 256 channels each, yielding a channel separation of $\sim 10 \mathrm{~km} \mathrm{~s}^{-1}$. We binned the channels further to obtain a final channel separation of $\sim 20 \mathrm{~km} \mathrm{~s}^{-1}$. The central position of the galaxy and four positions at a distance of one HPBW $\left(9.3^{\prime}\right)$ from the galaxy center to the NW, SW, SE, and NE, respectively, were observed in on-off mode using pairs of $5 \mathrm{~min}$ on source and $5 \mathrm{~min}$ off source integrations. The total integration times are shown in Table 2. Care was taken to avoid other Virgo galaxies with radial velocities within our bandwidth in all 5 on and off source positions. We used 3C 286 for pointing and flux calibration. The on and off observation times ranged between $80 \mathrm{~min}$ and $120 \mathrm{~min}$ per position. The resulting noise (Table 2), which is partly determined by small amplitude interferences, is close to the theoretical noise of $2 \mathrm{mJy}$ per hour of integration.

\section{Results}

The 5 Effelsberg spectra are shown in Figs. 2 and 11. In order to compare our single-dish HI spectra to interferometric data, where the galaxy is spatially resolved, we use the VLA $21 \mathrm{~cm} \mathrm{C}$ and D array data of Phookun et al. (1993). These data have a spatial resolution of $32^{\prime \prime} \times 28^{\prime \prime}$ and a channel separation of $10 \mathrm{~km} \mathrm{~s}^{-1}$. The bulk of the HI emission detected with the VLA is contained within a $\sim 6^{\prime} \times 5^{\prime}$ area, while a faint blob of HI was found $\sim 3^{\prime}$ south-east of the galaxy center and a very faint HI tail was detected towards the north-west, extending out to $10^{\prime}$ from the center.

In order to enhance the diffuse and extended HI emission, we smoothed the data cube to a resolution of $2^{\prime}, 3^{\prime}, 4^{\prime}$, and $5^{\prime}$.
Table 2. Integration times and rms.

\begin{tabular}{lccccc}
\hline \hline Position & Center & Northwest & Southwest & Southeast & Northeast \\
\hline$\Delta t(\min )$ & 60 & 60 & 60 & 40 & 45 \\
rms (mJy) & 2.8 & 1.8 & 1.4 & 3.5 & 1.7 \\
\hline
\end{tabular}

Three-dimensional masks were produced by setting all pixels to one where the intensity is above $5 \sigma$ and zero otherwise. This mask was applied to the initial data cube. For the 5 positions, synthesized spectra from the VLA data were produced using the position of the pointing and the beamsize of the Effelsberg $100-\mathrm{m}$ telescope at $21 \mathrm{~cm}\left(9.3^{\prime}\right)$. We thus use the spatial information of the VLA data to construct the synthesized spectra. It turned out that a smoothing to a $4^{\prime}$ resolution leads to a maximum flux density in the spectra. Therefore, all synthesized spectra are made with a $4^{\prime}$ smoothing. Moment maps were made of the masked cube without clipping (Fig. 1). In these maps the north-western, very low surface density plume is well detected.

The resulting spectrum for the central position is represented by the dashed line in the lower panel of Fig. 11. We find a total single-dish flux of $67.7 \mathrm{Jy} \mathrm{km} \mathrm{s}^{-1}$, which corresponds to an atomic gas mass of $4.6 \times 10^{9} M_{\odot}$. The central velocity is $2406 \pm 10 \mathrm{~km} \mathrm{~s}^{-1}$, the linewidths at $50 \%$ and $20 \%$ of the peak flux are $W_{50}=230 \mathrm{~km} \mathrm{~s}^{-1}$ and $W_{20}=270 \mathrm{~km} \mathrm{~s}^{-1}$. The total and peak flux densities of Phookun et al. (1993) are within $\sim 10 \%$ of our values, which is within the calibration accuracy. Both spectra agree reasonably well.

Figure 2 shows the $100-\mathrm{m}$ spectra of the 4 off-center positions (solid lines), together with the synthesized VLA spectra. The NE and SE Effelsberg and VLA spectra are in very good agreement. The Effelsberg NW spectrum shows excess emission between -100 and $0 \mathrm{~km} \mathrm{~s}^{-1}$ and less emission between 0 and $100 \mathrm{~km} \mathrm{~s}^{-1}$ than the VLA spectrum. The Effelsberg SW spectrum shows excess emission around $-200 \mathrm{~km} \mathrm{~s}^{-1}$ and less emission between 0 and $100 \mathrm{~km} \mathrm{~s}^{-1}$ than the VLA spectrum. These differences can be explained by three effects: (i) pointing errors of the Effelsberg telescope; (ii) disk emission detected by the sidelobes of the Effelsberg telescope; and (iii) extended $\left(>15^{\prime}\right)$ and/or very low surface density $(<2 \times$ $10^{18} \mathrm{~cm}^{-2}$ ) gas which was not detected by the VLA. The excess emission of the NW and SW VLA spectra in the velocity range between 0 and $100 \mathrm{~km} \mathrm{~s}^{-1}$ were possibly due to pointing errors of the Effelsberg telescope. A pointing error of about $10 \%$ of the telescope's HPBW to the west can account for the VLA excess emission with respect to the Effelsberg spectrum. However, such a pointing error is unlikely, though in principle not impossible, as it is 3 to 5 times the typical pointing correction, we had to apply during the observing run. It is not excluded that the Effelsberg excess emission with respect to the VLA spectrum is due to a sidelobe detection of the disk emission of the galaxy. The Effelsberg antenna diagram shows 4 first-sidelobe maxima at a distance of $\sim 13^{\prime}$ from the beam center and at a $-20 \mathrm{db}$ level ( $1 \%$ of the peak). One percent of the emission of the central profile (Fig. 11) is $\sim 3 \mathrm{mJy}$, which corresponds approximately to the $3 \sigma$ noise levels indicated in 

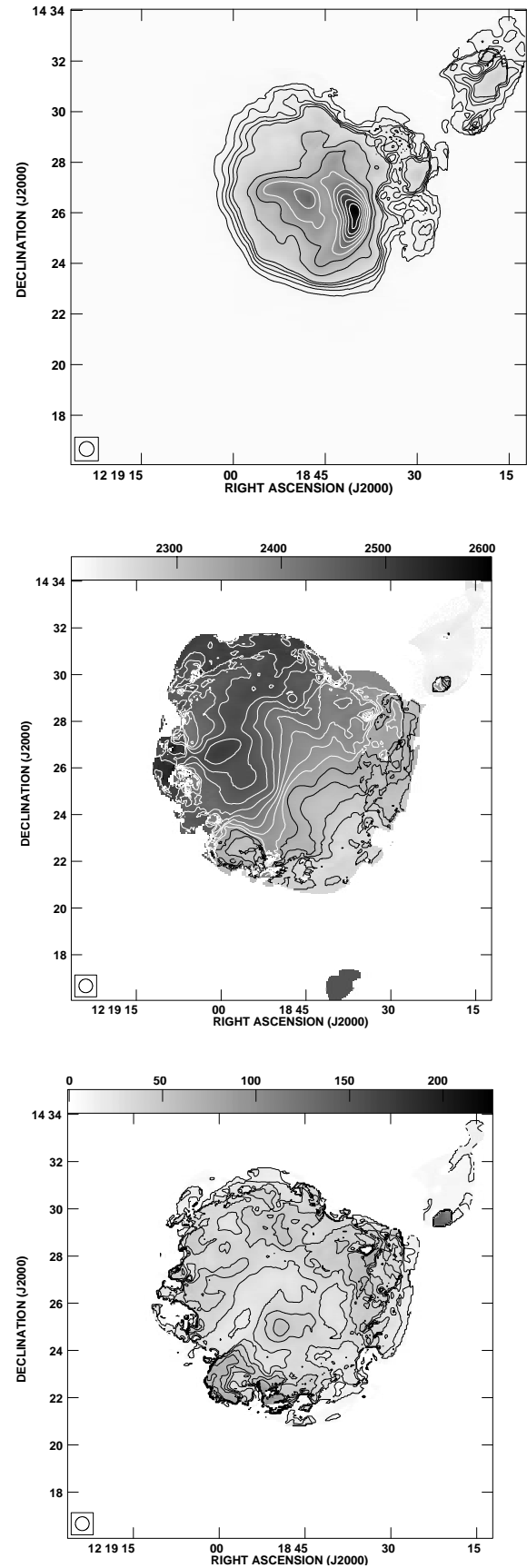

Fig. 1. HI observations of NGC 4254 (Phookun et al. 1993). Masks were produced from the data cube, which was smoothed to a resolution of $4^{\prime}$. The spectra were integrated within these masks. Top panel: HI distribution. The contour levels in all images are $(0.02$, $0.04,0.06,0.08,0.1,0.2,0.3,0.4,0.5,0.6,0.7,0.8,0.9,1.0)$ times the maximum of the image. Middle panel: velocity field. The contour levels of all images are at $2250-2510 \mathrm{~km} \mathrm{~s}^{-1}$ at intervals of $20 \mathrm{~km} \mathrm{~s}^{-1}$. Bottom panel: velocity dispersion. The contour levels in all images are at $10-60 \mathrm{~km} \mathrm{~s}^{-1}$ in steps of $10 \mathrm{~km} \mathrm{~s}^{-1}$.

the NW and SW spectra. Indeed, an inspection of the parallactic angle of the Effelsberg observations showed that its variation was small for the eastern positions, where the galaxy was located near a minimum of the sidelobes, whereas the variation was larger for the SW position and maximum for the
Table 3. Parameters of the different galactic components.

\begin{tabular}{llll}
\hline \hline Component & $N$ & $M\left(M_{\odot}\right)$ & $l(\mathrm{pc})$ \\
\hline halo & 16384 & $9.2 \times 10^{6}$ & 1200 \\
bulge & 16384 & $3.2 \times 10^{5}$ & 180 \\
disk & 16384 & $1.6 \times 10^{6}$ & 240 \\
companion & 11000 & $9.2 \times 10^{6}$ & 1200 \\
\hline
\end{tabular}

NW position, where the maximum of the sidelobe passed over the galactic disk.

In conclusion, the differences between the western Effelsberg and VLA spectra can be explained in principle by a mixture of effects (i), (ii), and (iii). However, we were not able to sort out which effect dominates. The northeastern position only shows disk emission, whereas the southwestern and southeastern positions contain additional low surface density, low velocity gas (at $\sim-200 \mathrm{~km} \mathrm{~s}^{-1}$ ). The most notable difference between the spectra occurs at the northwest position, where the Effelsberg and VLA spectra show three peaks: one due to galactic rotation at high velocities, a major peak at $-50 \mathrm{~km} \mathrm{~s}^{-1}$, and a third distinct peak at $\sim-180 \mathrm{~km} \mathrm{~s}^{-1}$. At the last two velocities, gas clumps were detected by Phookun et al. (1993) in the northwestern tail. Our spectrum reveals a bimodal velocity structure of the extended low surface density gas tail, which is already present, though less broad, in the VLA synthesized spectrum. We find a total line flux of $3.1 \mathrm{Jy} \mathrm{km} \mathrm{s}^{-1}$ $\left(2.1 \times 10^{8} M_{\odot}\right)$ or $4.6 \%$ of the total HI flux in the extended tail, more than the $3 \%$ of the total flux found by Phookun et al. (1993).

\section{The model}

Since the model we used is described in detail in Vollmer et al. (2001) and Vollmer (2003), we will only briefly summarize its main features here. The particles represent gas cloud complexes which are evolving in an analytically given gravitational potential of the galaxy.

The non-collisional component of the NGC 4254 model consists of 49125 particles, which simulate the galactic halo, bulge, and disk. The characteristics of the different galactic components shown in Table 3 are the number of particles $N$, the particle mass $M$, and the smoothing length $l$. The particle trajectories are integrated using an adaptive timestep for each particle as described in Springel et al. (2001) and applying the following criterion for an individual timestep:

$\Delta t_{i}=\frac{20 \mathrm{~km} \mathrm{~s}^{-1}}{a_{i}}$,

where $a_{i}$ is the acceleration of particle $i$. The minimum of all $\Delta t_{i}$ defines the global timestep used for the Burlisch-Stoer integrator that integrates the collisional component.

The initial conditions setup was made using the program described in Hernquist (1993) with the following parameters:

- halo: mass $M=1.5 \times 10^{11} M_{\odot}$, core radius $r_{\mathrm{c}}=3 \mathrm{kpc}$, tidal radius $r_{\mathrm{t}}=30 \mathrm{kpc}$, cutoff radius $R=90 \mathrm{kpc}$; 

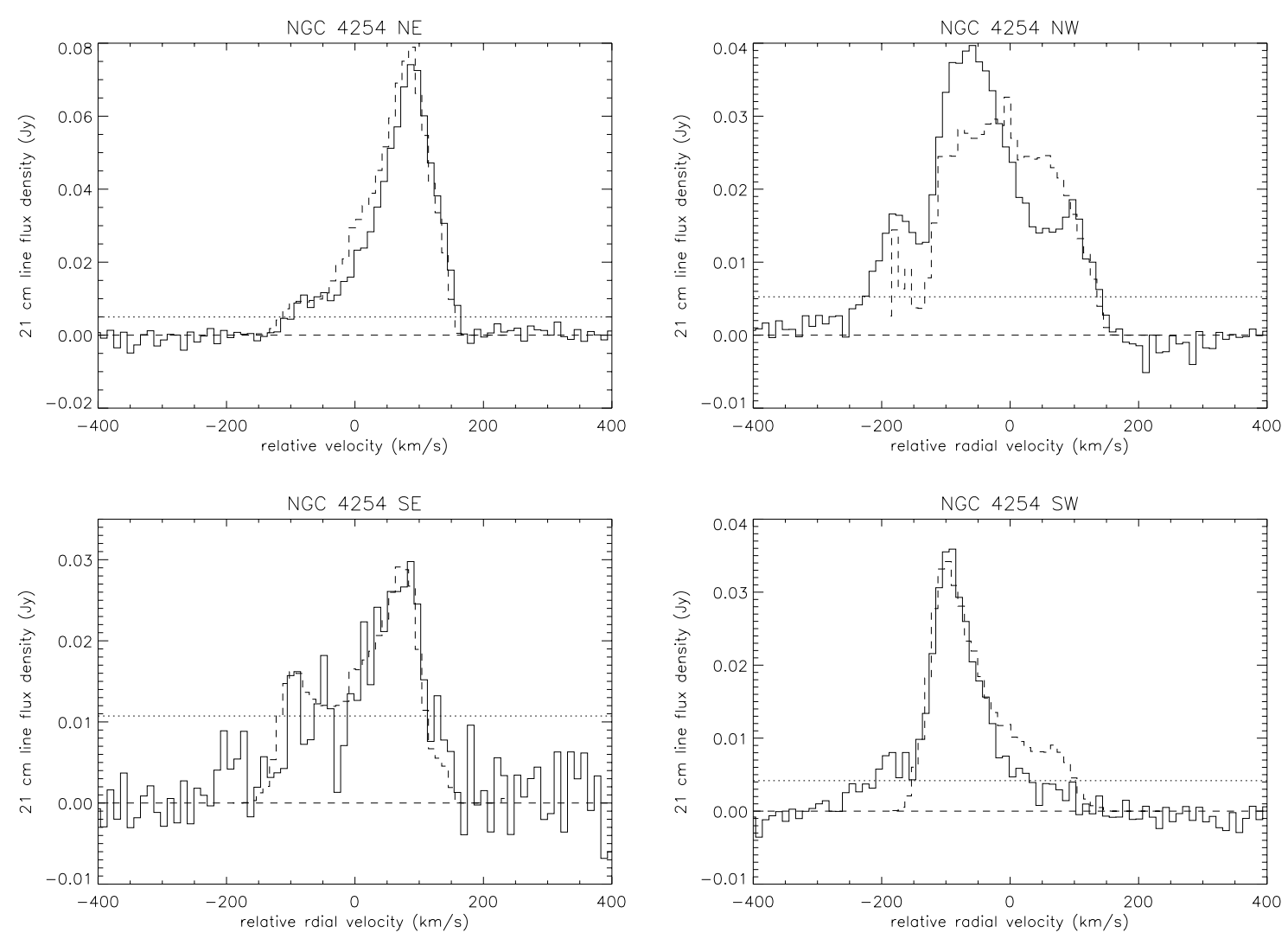

Fig. 2. Solid lines: Effelsberg 100-m spectra of the four off-center positions. Their locations with respect to the galaxy center are marked on top of each panel. Dashed line: synthesized VLA spectra. Dotted line: $3 \sigma$ noise levels of the Effelsberg spectra. Velocities are given relative to the systemic velocity of NGC 4254, $2407 \mathrm{~km} \mathrm{~s}^{-1}$.

- bulge: mass $M=5.2 \times 10^{9} M_{\odot}$, scale length $l=0.3 \mathrm{kpc}$, cutoff radius $R=30 \mathrm{kpc}$;

- disk: mass $M=2.6 \times 10^{10} M_{\odot}$, scale length $l=3 \mathrm{kpc}$, cutoff radius $R=45 \mathrm{kpc}$, disk thickness $z_{0}=600 \mathrm{pc}$.

The collisional component of the model consists of 20000 model particles representing gas cloud complexes of different masses that are rotating within the gravitational potential of the galaxy. The total gas mass is $M_{\text {gas }}^{\text {tot }}=5.8 \times$ $10^{9} M_{\odot}$, which is $\sim 30 \%$ larger than the observed gas mass (Phookun et al. 1993). To each particle a radius is attributed depending on its mass. During the disk evolution the particles can have inelastic collisions, the outcome of which (coalescence, mass exchange, or fragmentation) is simplified following Wiegel (1994). This results in an effective gas viscosity in the disk.

As the galaxy moves through the intracluster medium (ICM), its clouds are decelerated by ram pressure. Within the galaxy's inertial system its clouds are exposed to a wind coming from the opposite direction of the galaxy's motion through the ICM. The temporal ram pressure profile has the form of a Lorentzian, which is realistic for galaxies on highly eccentric orbits within the Virgo cluster (Vollmer et al. 2001). The effect of ram pressure on the clouds is simulated by an additional force on the clouds in the wind direction. Only clouds that are not protected by other clouds against the wind are affected.
To model NGC 4254, the 20000 collisional particles with a $1 / R$ column density profile were added to the 49125 noncollisional particles, and the system was evolved during $2 \mathrm{Gyr}$ in order to let it reach a relaxed state. At the end of the simulation the difference between the total energy and the total angular momentum of the system was smaller than $0.5 \%$ of their initial values. During the last Gyr of this simulation the disk properties (disk scale height, surface density profile, and density profile) changed less than $5 \%$. The final state of this simulation was used as the initial state for the simulations presented here.

The companion galaxy with a mass of $10^{11} M_{\odot}$ is simulated by 11000 non-collisional particles forming a Plummer sphere with a core radius of $2 \mathrm{kpc}$.

\section{The simulations}

\subsection{The tidal interaction}

We have modeled a close and rapid encounter between NGC 4254 and another massive Virgo cluster galaxy, for which we will try to identify a possible candidate at the end of this section. The closest distance between the two galaxies is $D=27 \mathrm{kpc}$, which occurs at $t=300 \mathrm{Myr}$ with a velocity difference of $710 \mathrm{~km} \mathrm{~s}^{-1}$. Snapshots of this tidal interaction are shown in Fig. 3. The position and inclination angle of NGC 4254 are used. The azimuthal angle is fixed in 

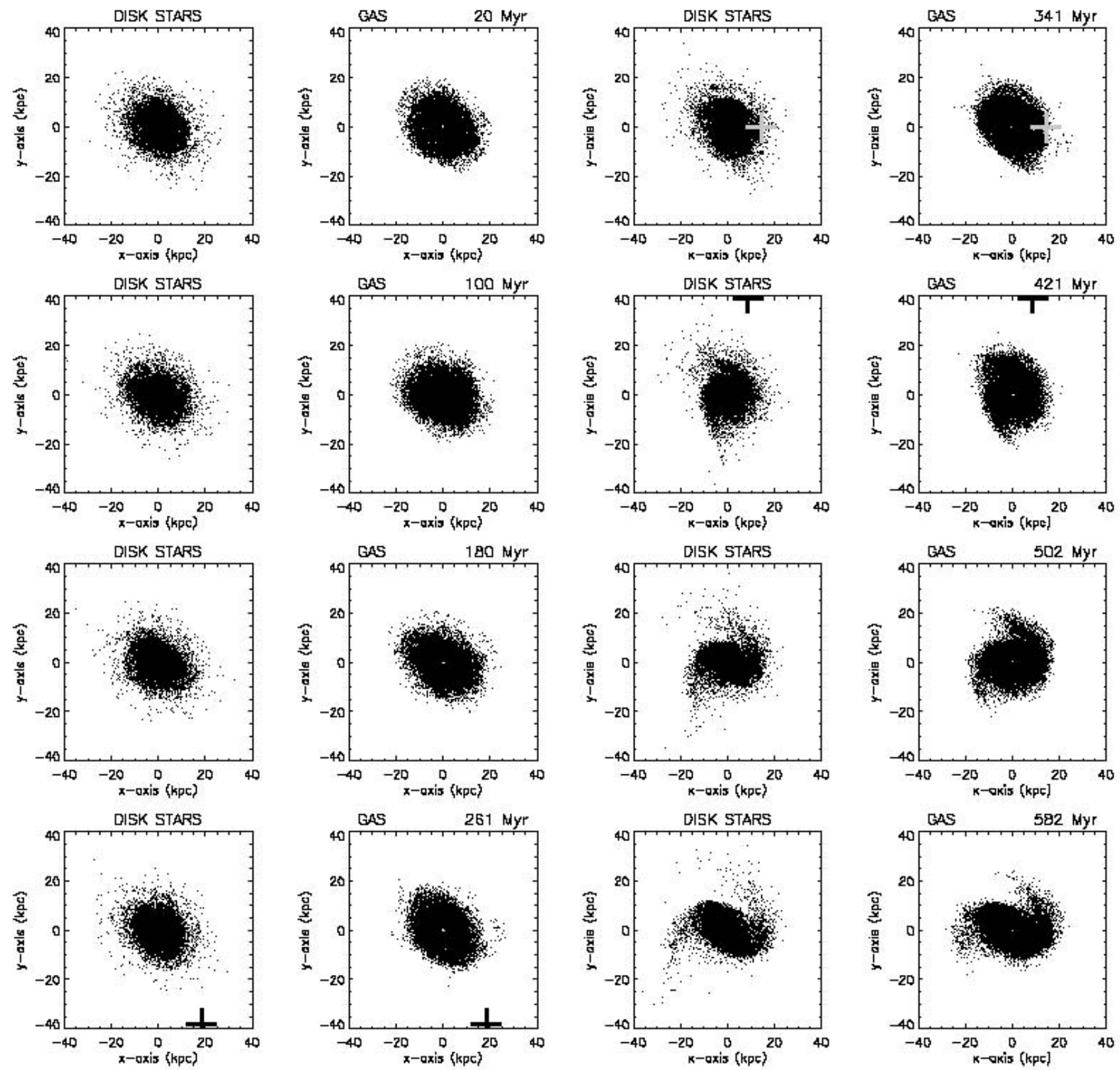

Fig. 3. Snapshots of the evolution of the NGC 4254 system model, undergoing only a tidal interaction with another massive Virgo cluster galaxy. The left panels show the stellar disk; the right panels show the gas. The timesteps are marked on the upper right of each panel showing the gas. The center position of the perturbing galaxy is marked as a cross. An animation of this simulation can be found in the electronic version.

a way to reproduce the observed spiral structure. The galaxy rotates clockwise. NGC 4254 starts building a spiral structure $\sim 100 \mathrm{Myr}$ after the closest passage of the perturbing galaxy. The spiral arm at the western side, where the interaction happened, is more prominent than the one at the opposite side. After $t=500 \mathrm{Myr}$ an asymmetric spiral pattern is formed with a prominent $m=1$ mode.

The final location of the perturbing galaxy in our simulation is $9 \mathrm{kpc}$ east and $120 \mathrm{kpc}$ north of the center of NGC 4254, at which point its radial velocity is $-550 \mathrm{~km} \mathrm{~s}^{-1}$ with respect to the systemic velocity of NGC 4254. A possible candidate is the SB0 galaxy NGC 4262, which is located $51 \mathrm{kpc}$ east and $137 \mathrm{kpc}$ north of NGC 4254. Its radial velocity is $\sim-1050 \mathrm{~km} \mathrm{~s}^{-1}$ with respect to the systemic velocity of NGC 4254. NGC 4262 has a ring of HI around it with twice the diameter $D_{25}$ of the optical disk and an inclination at least $50^{\circ}$ with respect to the plane of the optical disk (Krumm et al. 1985). With an HI rotation velocity of $220 \mathrm{~km} \mathrm{~s}^{-1}$ at $2^{\prime}$ radius its total mass is near the $10^{11} M_{\odot}$ used in our model. Since NGC 4262 is an S0 galaxy and therefore much more concentrated than a spiral galaxy, we do not expect to observe an impact of the interaction on its stellar content (see also NGC 4435 in the NGC 4438/NGC 4435 system, Combes et al. 1988). Concerning the HI distribution of NGC 4262, outer ring structures are frequently observed in gas-rich S0 galaxies; see e.g. van Driel \& van Woerden (1991), who consider that, in general, their origins are most likely a galaxy merger or a star formation burnt-out scenario. 
Among the S0 galaxies studied by van Driel \& van Woerden, NGC 4262 has by far the highest inclination difference $\left(60^{\circ}\right)$ between the planes of its outer HI ring and the stellar disk. For NGC 4262 we can only speculate that the interaction with NGC 4254 is at least partly responsible for the peculiar ring structure. Its approximate symmetry is consistent with the time delay between the gravitational interaction and today, which is about one rotation period of the ring.

\subsection{Tidal interaction and ram pressure}

In a second step we introduce a temporal ram pressure profile of the form:

$p_{\text {ram }}=\frac{10 t_{1}^{2} \rho_{0} v_{0}^{2}}{\left(t-t_{0}\right)^{2}+t_{1}^{2}}$,

where $\rho_{0}=10^{-4} \mathrm{~cm}^{-3}, v_{0}=1000 \mathrm{~km} \mathrm{~s}^{-1}, t_{0}=1 \mathrm{Gyr}$, and $t_{1}=115$ Myr. Since the inclination angle between the disk and the galaxy's orbital plane within the cluster is $70^{\circ}$, the stripping occurs nearly face-on and is very efficient - see Vollmer et al. (2001) for a discussion of the stripping efficiency with respect to the inclination angle. While face-on stripping $\left(i>60^{\circ}\right)$, and thus a high stripping efficiency, is necessary to reproduce the observed northwestern, very low surface brightness plume, the inclination angle, on the other hand, must be smaller than $90^{\circ}$, i.e. face-on, to produce the northwest asymmetry of the plume. For these simulations the shape of the galaxy's orbit within the cluster does not play a dominant role. The important conditions are (i) that the galaxy is entering the cluster and (ii) that ram pressure at the time of interest is $p_{\text {ram }}(580 \mathrm{Myr})=0.7 \rho_{0} v_{0}^{2}$, consistent with its radial velocity and the ICM density derived from X-rays (Schindler et al. 1999).

However, when we run our simulations with a constant cloud column density of $\Sigma_{0}=10^{21} \mathrm{~cm}^{-2}$, this ram pressure does not produce a gas tail as observed for NGC 4254, while increasing the ram pressure to produce such a gas tail has too great an effect on the gas disk and pushes a considerable fraction of the southeastern part of the galaxy towards the northwest, which is not observed.

Therefore, we decided to add the effects of cloud expansion and evaporation to the numerical code: once the clouds are pushed out of the galactic disk by ram pressure, they may expand or evaporate within the hot intra-cluster medium (Cowie $\&$ McKee 1977). To simulate these effects, the column density of those clouds that are located in regions with a local density below a threshold $\rho_{\text {lim }}$ is made to decrease exponentially with time as $\Sigma=\Sigma_{0} \exp \left(-t / t_{2}\right)$, where $t_{2}=50 \mathrm{Myr}$. This corresponds approximately to the timescale of saturated evaporation (Vollmer et al. 2001). The threshold gas density is fixed at $\rho_{\text {lim }}=10^{-2} \mathrm{~cm}^{-3}$, and the parameters $t_{2}$ and $\rho_{\text {lim }}$ are chosen such that the HI properties of NGC 4254 are reproduced. Snapshots of the evolution of the NGC 4254 system undergoing a tidal interaction, as well as ram pressure stripping, are shown in Fig. 4. The position and inclination angle of NGC 4254 are used. The evolution of the stellar content is very similar to that in the previous simulation without ram pressure stripping (Fig. 4), because ram pressure selectively affects only the gas.
From the time of closest passage ( $t=300 \mathrm{Myr}$ ) onwards, there is an increase in the difference between the gas distribution in the tidal interaction simulation and the mixed interaction simulation. Since the gas clouds that are expelled from the galaxy by the tidal interaction have a lower local gas density, their column density decreases, and they are stripped easily by a moderate amount of ram pressure. This can be noted by the amount of low volume density gas that has been pushed towards the northwest at $t=400 \mathrm{Myr}$. In the forming western spiral arm, the high local gas density prevents the gas from being stripped, and only the gas located outside the arm is stripped.

\subsection{Comparison between observations and simulations}

We chose the snapshot at $t=580 \mathrm{Myr}$ for the comparison with the observations, because (i) it reproduces the spiral pattern of NGC 4254 and (ii) it shows a very low averaged surface density tail to the northwest as observed. At that moment the ram pressure is only $p_{\text {ram }}=0.7 \rho_{0} v_{0}^{2}=70 \mathrm{~cm}^{-3}\left(\mathrm{~km} \mathrm{~s}^{-1}\right)^{2}$. Using the Gunn \& Gott (1972) approximation we obtain

$\Sigma v_{\text {rot }}^{2} R^{-1}=p_{\text {ram }}$,

where $\Sigma$ is the gas surface density, $v_{\text {rot }}$ the rotation velocity of the galaxy, and $R$ the galactic radius. With $v_{\text {rot }}=170 \mathrm{~km} \mathrm{~s}^{-1}$ and $\Sigma=10^{20} \mathrm{~cm}^{-2}$ we obtain $R=14 \mathrm{kpc}$, or about the optical size $D_{25}$ of the galaxy. Thus ram pressure affects only gas with $\Sigma<10^{20} \mathrm{~cm}^{-2}$. Since the radius where the radially averaged $\Sigma=10^{20} \mathrm{~cm}^{-2}$ of the HI gas in NGC 4254 is $18 \mathrm{kpc}$ (Warmels et al. 1988; Cayatte et al. 1994), i.e. 1.2 times the optical radius, we do not expect the galaxy to be affected by ram pressure. We suggest that the peculiar low surface density gas distribution and kinematics are due to the interplay between the tidal interaction and ram pressure stripping. The observed and the simulated distribution of the stellar content are shown in Fig. 5. Like the observed main spiral pattern, the simulated spiral structure is asymmetric with a wider western spiral arm and a tighter eastern spiral arm. In addition, the observed spiral structure shows an $m=3$ and an $m=5$ mode, which are not reproduced by the model. The excitation of these modes depends crucially on the mass distribution within the galaxy. Since the aim of this study is not to reproduce the complicated spiral pattern of NGC 4254 exactly but its gas distribution and kinematics, we have not attempted to investigate the influence of different mass distributions on the spiral pattern, which would also require a larger number of particles.

\subsection{The moment maps}

For the moment maps of the HI data we smoothed the cube to a resolution of $35^{\prime \prime} \times 35^{\prime \prime}$ and clipped the data at a $0.8 \mathrm{mJy} /$ beam/channel level. A comparison between simulated and observed gas distributions at $t=580 \mathrm{Myr}$ is shown in Fig. 6. Since we do not differentiate between atomic and molecular hydrogen, the simulated gas distribution shows the total gas content. Although the model gas distributions are more concentrated in the central part of the galaxy than is observed in HI, it should be noted that in the central few kpc 



Fig. 4. Snapshots of the evolution of the NGC 4254 system model undergoing a tidal interaction with another massive Virgo cluster galaxy, as well as ram pressure stripping. The left panels show the stellar disk; the right panels show the gas. The timesteps are marked on the upper right of each panel showing the gas. The center position of the perturbing galaxy is marked as a cross. The arrow indicates the direction of ram pressure, and the length of the arrow is proportional to its strength. An animation of this simulation can be found in the electronic version.

of NGC 4254 most of the gas is molecular (Kenney \& Young 1988), which makes the total gas distribution resemble the simulations. In the simulations without ram pressure stripping, the gas follows the stellar spiral structure closely, as expected. This is also the case in the inner parts of the galaxy when ram pressure stripping is included, whereas the outer parts of the gas distribution show important differences, since ram pressure has pushed the gas from the southeastern quadrant towards the center. We suggest that the observed local HI maximum in the southeast is due to this effect. The low surface density northern extension is fairly well reproduced by the simulations including ram pressure stripping. However, this feature extends more to the west in the observed data. The observed northwestern very low surface density plume is present in the simulations including ram pressure stripping, but their surface density structures differ. While in the simulations there is a gradient to lower surface densities to the north, the observations show a sudden drop between the galactic main disk and the plume (see also Fig. 1). Within the plume the surface density seems to be rather constant. The observed southern low-surface density blob is not present in our simulations.

A comparison between the simulated and observed velocity fields at $t=580 \mathrm{Myr}$ is shown in Fig. 7. The effects of ram pressure stripping on the velocity field are the following: (i) on the eastern side of the galaxy the gas with low radial velocities is pushed towards the northwest, and some of the gas is 

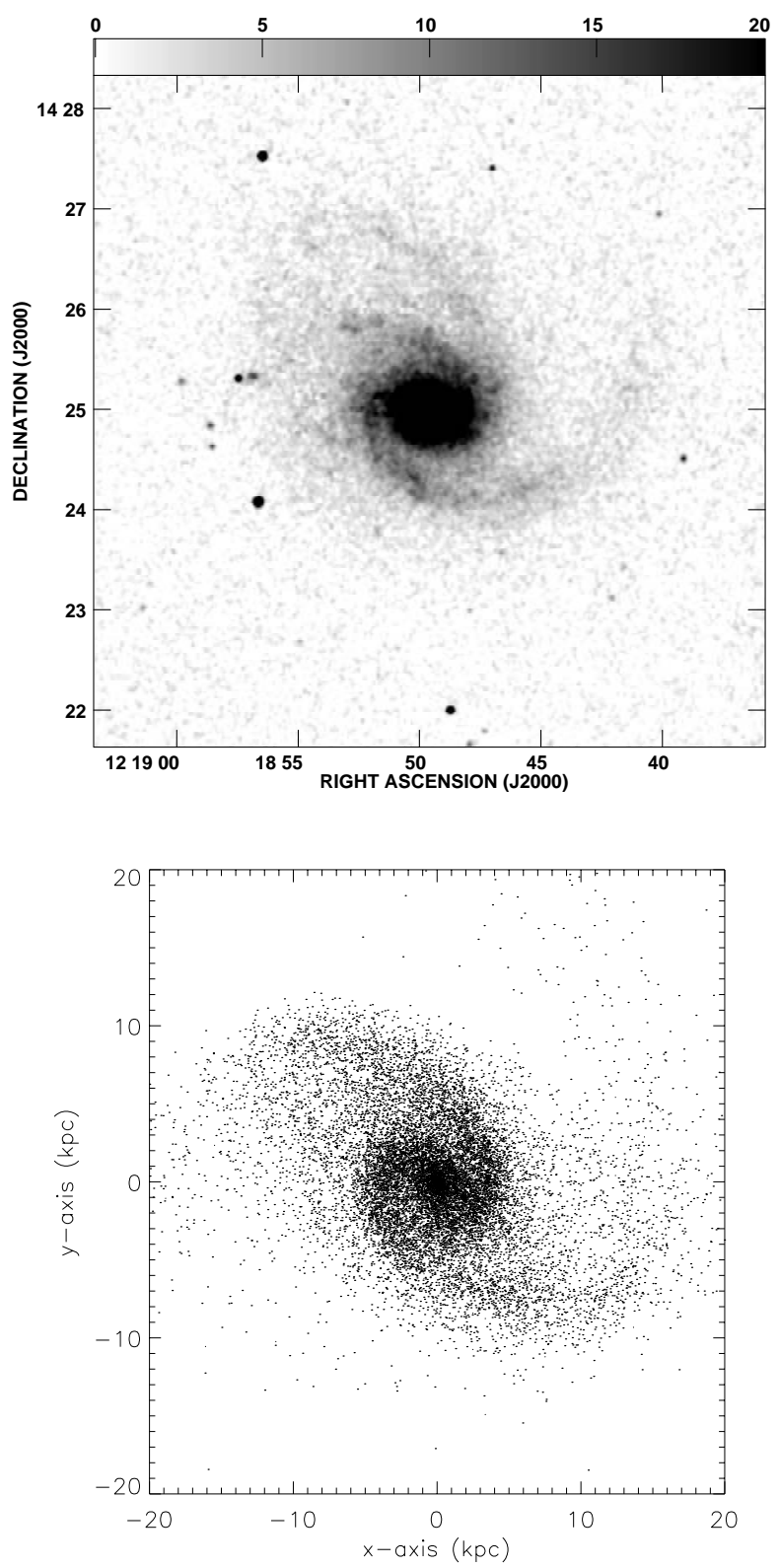

Fig. 5. The stellar component of NGC 4254. Upper panel: 2MASS $K$ band image (from NED). Lower panel: model distribution for the model with both tidal interaction and ram pressure stripping; snapshot at $t=580 \mathrm{Myr}$.

decelerated towards the cluster mean velocity $\left(1100 \mathrm{~km} \mathrm{~s}^{-1}\right)$. The net effect is that the eastern maximum is displaced to the south and has a narrower shape; (ii) the western plateau of the rotation curve is less pronounced when ram pressure stripping is added to the simulations; (iii) the velocity contours are straightened to the north, but the imprint of the spiral arm's velocity structure is still visible. This velocity structure is more pronounced in the observed velocity field. The straightening of the model velocity contours to the north can be observed in Fig. 1. The observed negative velocities with respect to the systemic velocity at $\mathrm{RA}=12^{\mathrm{h}} 18^{\mathrm{m}} 50^{\mathrm{s}}$ and $\mathrm{Dec}=14^{\circ} 28^{\prime} 00^{\prime \prime}$ do not have counterparts in the simulations.
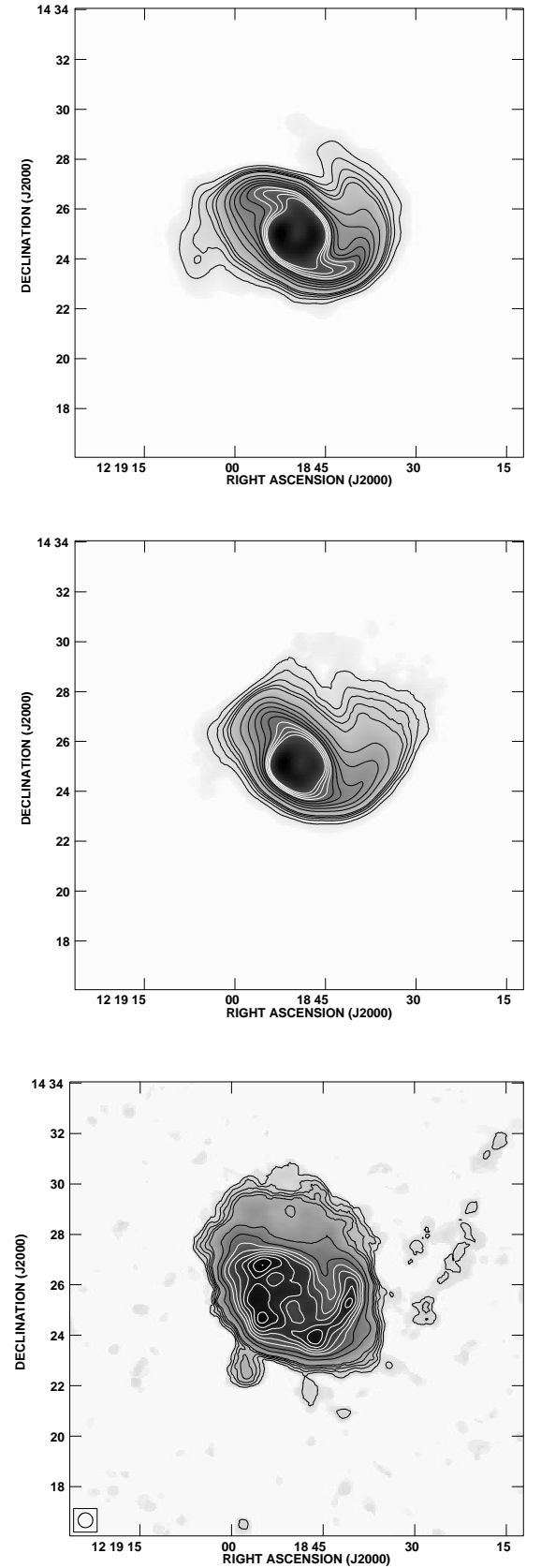

Fig. 6. The gaseous component of NGC 4254. Upper panel: model distribution of the HI gas for the model with only a gravitational interaction. Middle panel: model distribution of the HI gas for the model with gravitational interaction plus ram pressure stripping. Lower panel: HI distribution of NGC 4254 observed by Phookun et al. (1993). The contour levels in all images are $(0.02,0.04,0.06,0.08,0.1$, $0.2,0.3,0.4,0.5,0.6,0.7,0.8,0.9,1.0$ ) times (i) half of the maximum of the image for the models and (ii) the maximum of the image for the Hi data.

The comparison between simulated and observed velocity dispersions at $t=580 \mathrm{Myr}$ are shown in Fig. 8. The velocity dispersion of the simulations without ram pressure stripping only shows a significant enhancement in the galaxy center, as expected. When ram pressure stripping is included, three additional local maxima are found: (i) to the east; (ii) to the north; and (iii) to the west of the center. The observed velocity 

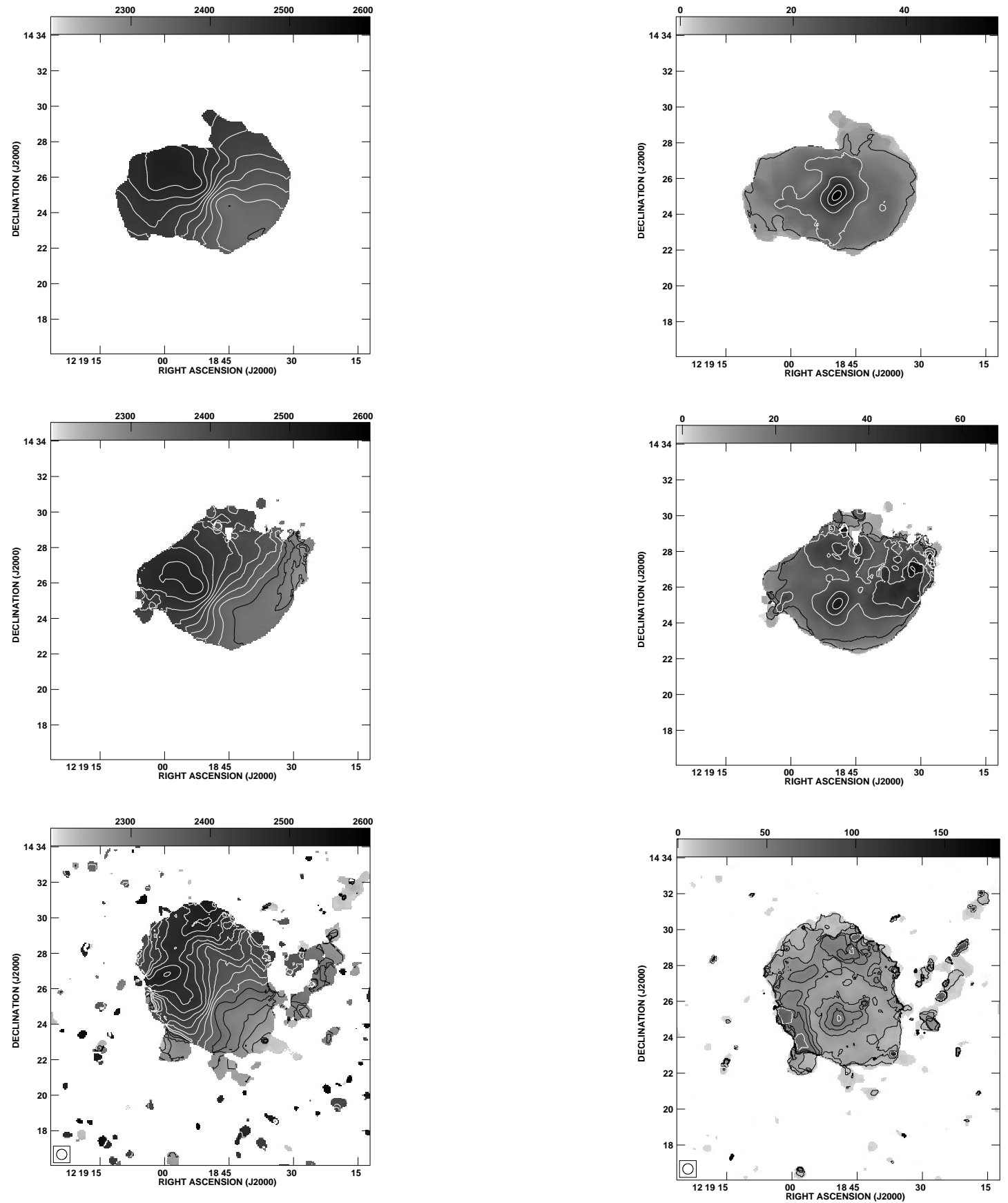

Fig. 7. Top panel: model distribution of the HI velocity field for a model of a gravitational interaction alone. Middle panel: distribution of the HI velocity field of the model of a gravitational interaction together with ram pressure. Bottom panel: HI velocity field of NGC 4254 (Phookun et al. 1993). The contour levels of all images are at $2250-2510 \mathrm{~km} \mathrm{~s}^{-1}$ at intervals of $20 \mathrm{~km} \mathrm{~s}^{-1}$.

dispersions also show extended maxima to the east, north, and west of the galaxy center (Fig. 1).

\subsection{The three dimensional data}

Since the three-dimensional HI distribution of NGC 4254 has a complex structure, moment maps do not show all the information contained in the data cubes. To complete the information contained in the moment maps, we show some selected

Fig. 8. The gaseous velocity dispersion in NGC 4254. Upper panel: model gas velocity dispersion map for the model with only a gravitational interaction. Middle panel: model gas velocity dispersion map for the model with both gravitational interaction and ram pressure stripping. Lower panel: HI velocity dispersion map (Phookun et al. 1993). The contour levels in all images are at $10-60 \mathrm{~km} \mathrm{~s}^{-1}$ in steps of $10 \mathrm{~km} \mathrm{~s}^{-1}$.

position-velocity diagrams, as well as a three dimensional visualisation of the model and observed HI cubes.

Figure 9 shows the HI data and the model including a gravitational interaction and ram pressure in 3 position-velocity diagrams along the declination axis and 3 others along the right ascension axis. The HI data is represented by contours and the model by the greyscale. The observed main features are 



Fig. 9. Position-velocity diagrams of 6 selected planes. Contours: HI observations (Phookun et al. 1993). Greyscale: model including ram pressure. Dark regions correspond to regions of low intensity.

all qualitatively reproduced. However, the low velocities of the low intensity southeastern edge $\left(\Delta \delta=-120^{\prime \prime}, 0^{\prime \prime} ; \Delta \alpha=160^{\prime \prime}\right)$ are not present in the model. We speculate that this represents gas initially located at the tip of the spiral arm that is decelerated by ram pressure to smaller velocities. However, a captured dwarf scenario might also be possible, which affects the gas kinematics locally. No optical counterpart is detected at this location. In addition, the model gas distribution at the northern edge $\left(\Delta \delta=200^{\prime \prime}\right)$ is offset from the observed gas distribution, as can be seen in Fig. 6.

A three-dimensional visualisation of the full data set is shown in Fig. 10, where the upper and middle panels represent, respectively, the final snapshots of the simulations (i) with a gravitational interaction alone (cf. Fig. 3) and (ii) with a gravitational interaction and ram pressure (cf. Fig. 4). The lower panel shows the HI data cube of Phookun et al. (1993) with an isosurface at $2 \mathrm{mJy} / \mathrm{beam} / \mathrm{channel}$. The two tidally induced spiral arms are clearly visible to the left and right of the upper panel. By comparing the upper and lower panels, the effects of ram pressure on the model gas distribution can be seen directly: the extent of the gas distribution in right ascension is decreased (cf. Fig. 6), the velocities in the high velocity arm are increased (the gas is accelerated; the wind and galactic rotation have the same direction), and those in the low velocity arm are decreased (the gas is decelerated; the wind and galactic rotation have opposite directions). These features can be qualitatively recognised in the HI data cube (Fig. 10, lower panel). In addition, the quasi horizontal edge at $v \sim 2460 \mathrm{~km} \mathrm{~s}^{-1}$ to the right of Fig. 10 (upper panel) is stretched to higher velocities in Fig. 10 (middle panel) due to the gas acceleration by ram pressure. However, we note that the observed gas velocities in Fig. 10 (lower panel) at that location in the data cube are shifted to much higher velocities $\left(\Delta v \sim 100 \mathrm{~km} \mathrm{~s}^{-1}\right)$. Moreover, the low velocities in the HI blob located in the upper left quadrant of Fig. 10 (lower panel), which corresponds to the southern blob of Fig. 6, are not reproduced. If we lower the intensity level of Fig. 10 (middle panel), single gas clouds become visible in this region of the cube, but they do not form an elongated structure as observed.

Thus we conclude that although the observed main features are reproduced qualitatively, there are quantitative differences between observations and simulations. However, we note that effects of ram pressure definitely improve the fit to the HI observations (gas displacement, acceleration, and deceleration). Only the amplitude of the mechanism is not strong enough to reproduce the $\mathrm{HI}$ in the lower right and upper left quadrant of Fig. 10 (lower panel). We speculate that this is mainly due to the missing physics of $\mathrm{HI}$ cloud expansion and evaporation in the model and, in a less important way, to the amount of ram pressure and the inclination angle between the disk and the galaxy's orbital plane within the cluster.

\subsection{The spectra}

The observed and simulated HI spectra of the main body of the HI distribution (see Sect. 3) are shown in Fig. 11. While all 


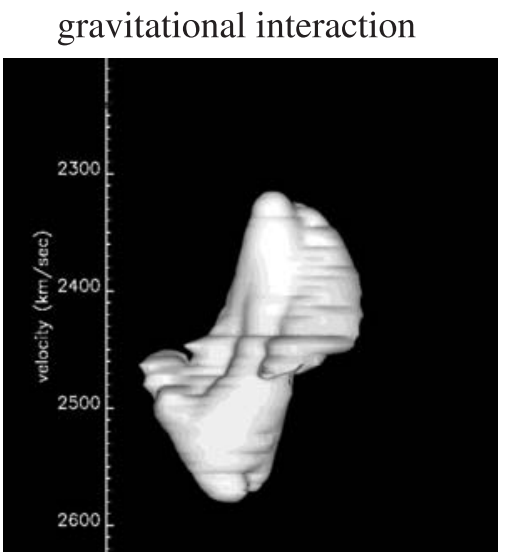

grav. interaction+ram pressure



HI data cube

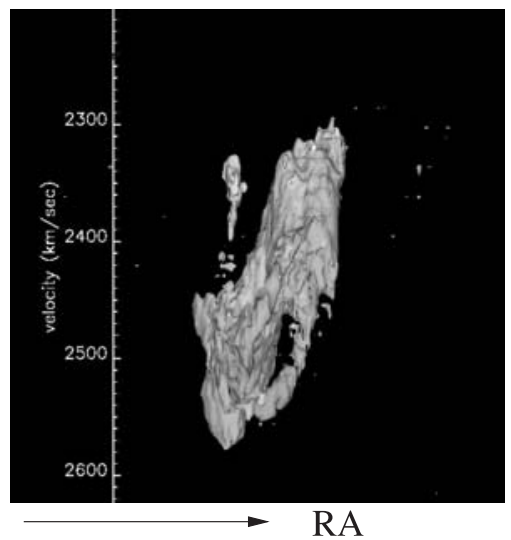

Fig. 10. 3D representation of data cubes. The axis of right ascension is indicated. The axis of declination is perpendicular to the image plane. Upper panel: model cube of the simulation including a gravitational interaction alone. Middle panel: model cube of the simulations including a gravitational interaction and ram pressure. Lower panel: observed HI data cube (Phookun et al. 1993). The intensity isosurface is at $2 \mathrm{mJy} / \mathrm{beam} / \mathrm{channel}$.

spectra show the characteristic double-horned structure, only the simulation that includes ram pressure stripping reproduces the observed tail at low velocities.

The observed and simulated HI spectra of the 4 off-center positions (see Sect. 3) are shown in Fig. 12 for the model with
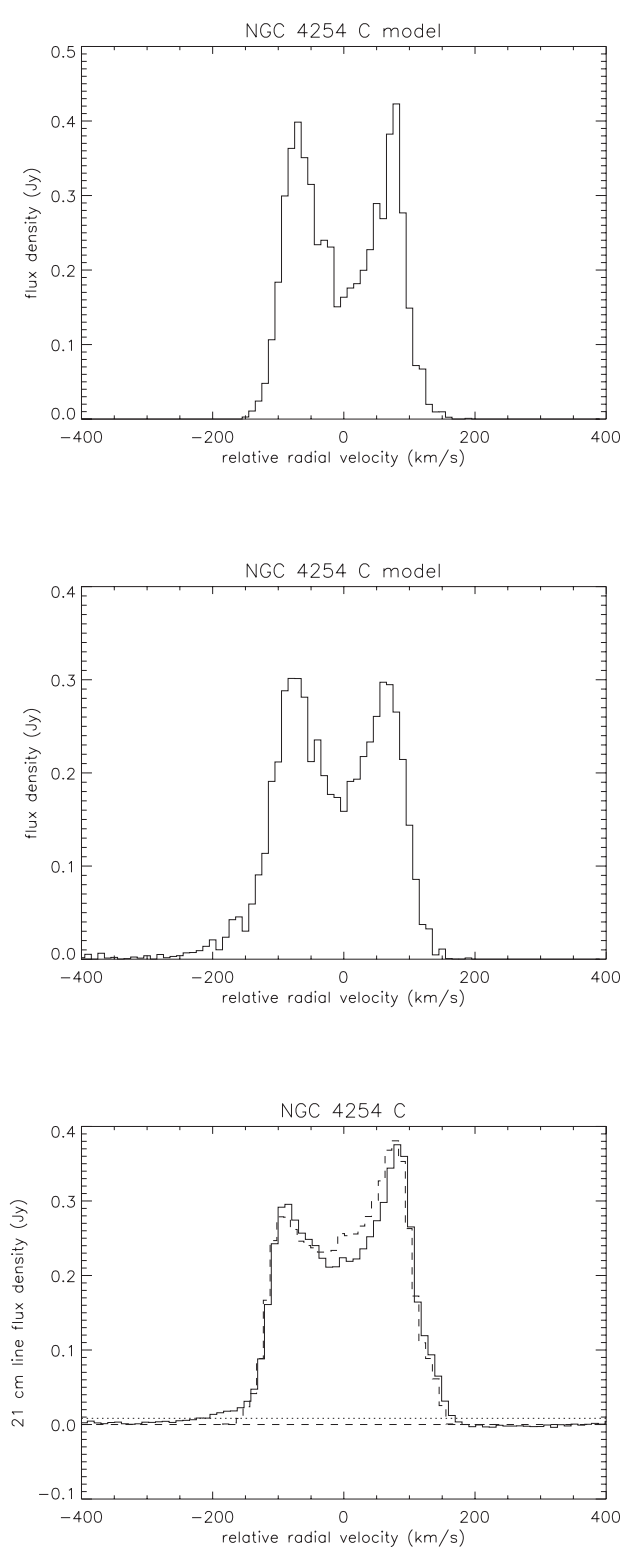

Fig. 11. The central HI spectrum of NGC 4254. Upper panel: simulated central Hi spectrum at $t=580 \mathrm{Myr}$ for the model with only a gravitational interaction. Middle panel: simulated central HI spectrum at $t=580 \mathrm{Myr}$ of the model with both gravitational interaction and ram pressure stripping. Lower panel: observations. Solid lines: Effelsberg 100-m single dish spectra of the four off-center positions. Their locations with respect to the galaxy center are marked on top of each panel. Dashed line: synthesized VLA spectrum. Dotted line: $3 \sigma$ noise level of the $100-\mathrm{m}$ spectrum. Radial velocities are given relative to the systemic velocity of NGC 4254 .

only gravitational interaction and in Fig. 13 for the model with both gravitational interaction and ram pressure stripping.

In the model without ram pressure stripping the spectrum at the NE position can already be reproduced well. The shift of the observed HI spectrum to higher velocities at $v=$ $100-150 \mathrm{~km} \mathrm{~s}^{-1}$ is due to the fact that the model does not reproduce the high velocities of the northern HI arm (see Sect. 5.5). Although the shape of the SE spectrum is reproduced, the observed flux densities are 1/3 higher than the simulated ones. The simulated SW spectrum lacks the observed emission at low 


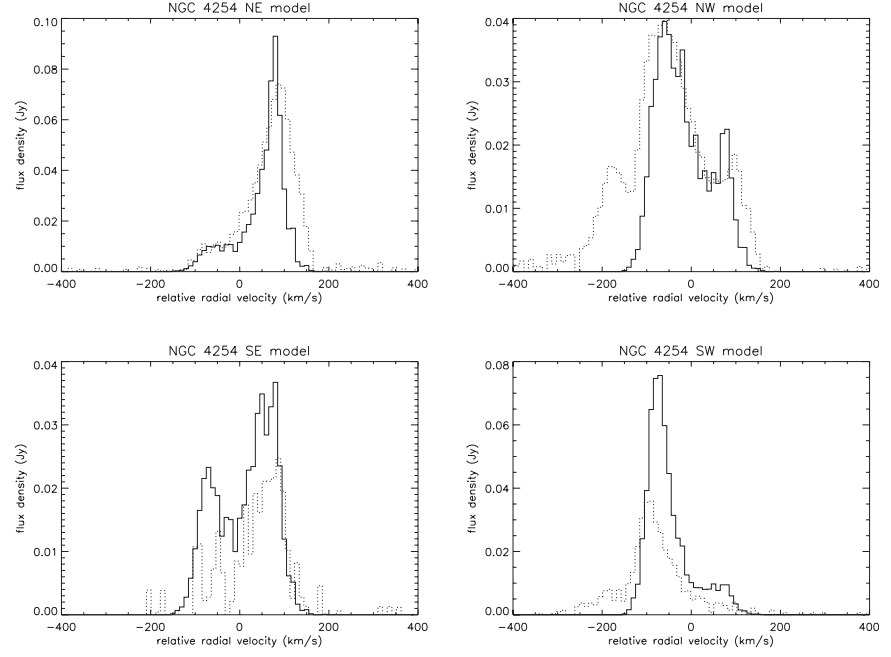

Fig. 12. Off-center positions HI spectra of NGC 4254. Solid line: simulated off-center spectra at $t=580 \mathrm{Myr}$ for the model with only a gravitational interaction. Dotted line: observed Effelsberg off-center spectra. The locations of the spectra with respect to the galaxy center (see Sect. 3) are marked on top of each panel. Radial velocities are relative to the systemic velocity of NGC 4254 .

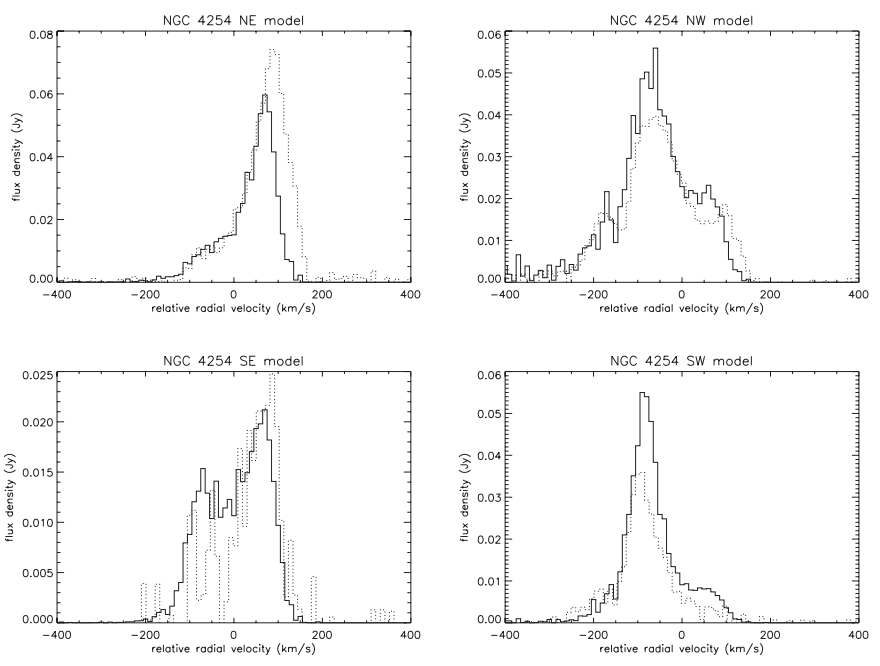

Fig. 13. Off-center positions HI spectra of NGC 4254. Solid line: simulated off-center spectra at $t=580 \mathrm{Myr}$ for the model with both gravitational interaction and ram pressure stripping. Dotted line: observed Effelsberg off-center spectra. The locations of the spectra with respect to the galaxy center (see Sect. 3) are marked on top of each panel. Radial velocities are relative to the systemic velocity of NGC 4254.

velocities, and the observed and simulated flux densities differ by a factor of two. The simulated NW spectrum completely lacks the observed peak at low velocities.

In the model that includes ram pressure stripping, the simulated NE spectrum is almost unchanged and reproduces the observations well. The simulated SE and SW spectra have lower flux densities than those for the simulations without ram pressure (Fig. 12) and give better fits to the observed spectra. The effects of ram pressure stripping on the NW spectrum are twofold: (i) the high-velocity maximum is decreased; and (ii) a low velocity tail appears - both effects are observed. Thus all 5 observed spectra are well, or, in some cases better, reproduced by the simulations including ram pressure stripping.

\section{Discussion}

Previously, two explanations were proposed for the $m=$ 1 mode of NGC 4254: (i) its intrinsic mass distribution that favors the formation of this spiral pattern (Iye et al. 1982); and (ii) the excitation of the $m=1$ mode by infalling HI gas together with a swing amplifier (Phookun et al. 1993). Based on our simulations we suggest another mechanism: a close and rapid tidal interaction that triggers an $m=1$ mode. Our models indicate that NGC 4254, when entering the Virgo cluster, had a close and rapid encounter with a $10^{11} M_{\odot}$ galaxy $\sim 280 \mathrm{Myr}$ ago, and has since then been undergoing increasing ram pressure stripping. The tidal interaction caused the $m=1$ spiral structure, and, together, the tidal interaction and ram pressure caused the peculiar HI distribution and kinematics. The intrinsic mass distribution of NGC 4254 then favors the formation of additional $m=3$ and $m=5$ spiral structures.

Phookun et al. (1993) discarded the ram pressure scenario on the following grounds: since NGC 4254 is moving towards the southeast, which makes the ram pressure push the gas located in the southeastern part of the galaxy towards the northwest, there can be no gas ahead of the galaxy as it moves into the intracluster medium. However, the observed low surface density gas blobs to the south of the main HI body represent such gas.

Although it is true that in an edge-on stripping scenario the existence of the southern low surface density gas blobs would rule out the ram pressure scenario, in our model stripping occurs almost face-on. In the case of a face-on stripping, the gas is removed in a cone-like structure and the projected radial distance of the stripped gas increases with its height from the disk (see Vollmer \& Huchtmeier 2003). This makes it in principle possible to observe low surface density gas seen in projection to the south of the main gas disk, which represents stripped gas that is actually located in front of NGC 4254 and which should show negative velocities with respect to the systemic velocity of NGC 4254 as is observed. The southern low surface density gas blobs thus do not contradict a face-on ram pressure stripping scenario, and we in fact find gas clouds in the south of the gas disk in the model snapshot that we compare to the observations in Fig. 4. These are not visible in the gas distribution (Fig. 6) and three-dimensional visualisation (Fig. 10), because their column density is very low.

Although our model with ram pressure stripping succeeds in reproducing the spiral structure and the overall gas distribution and kinematics, the main difference between our model and the observations is the shape of the extended low surface density gas tail observed to the northwest. While the simulated gas tail is a smooth continuation of the $m=1$ spiral arm, the observed tail consists of single blobs at the level of sensitivity of the VLA data, and there is a gap between the gas disk and the tail, which might have two reasons: (i) once the stripped gas leaves the disk, it is ionized and expanding through ICM-ISM mixing, and is thus no longer visible in the HI line. At a later stage it might cool and recombine again. (ii) The stripping is 
not continuous, but occurs in discrete events, because the ICM is not homogeneous. In this case the observed tail might represent gas falling back. We did not attempt to simulate this effect, because we do not have enough information on the temporal profile of such discrete events.

The evolutionary history of NGC 4254 thus closely resembles that of NGC 4654 (Vollmer 2003). Both galaxies had a close and rapid encounter with another massive Virgo cluster galaxy, and both galaxies are presently still undergoing ram pressure stripping. In both cases, the tidal interactions make the stripping more efficient. For NGC 4254 we had to include the effects of gas expansion and/or evaporation in the simulations, which was not necessary for NGC 4654. If we had included these effects in the NGC 4654 simulations, we could have used a ram pressure that increases with time and whose maximum value would have been a few times smaller.

Recently Minchin et al. (2005) detected $1.6 \times 10^{8} M_{\odot}$ of neutral hydrogen in the object VIRGOHI21, which has a radial velocity of $\sim 2000 \mathrm{~km} \mathrm{~s}^{-1}$ and lies $\sim 120 \mathrm{kpc}$ north-west from NGC 4254. The total HI flux of VIRGOHI21 is too low and its position too far away from the beam center of our NW position to be detectable in the Effelberg spectrum. We exclude that VIRGOHI21 represents tidal debris from NGC 4254 for two reasons: (i) the velocity gradient within VIRGOHI21 is opposite to the gradient between NGC 4254 and VIRGOHI21; and (ii) if VIRGOHI21 were tidal debris, the second interacting galaxy should be located roughly on an extrapolated line between NGC 4254 and VIRGOHI21, but there is no galaxy close enough in velocity in this region of the sky.

\section{Conclusions}

NGC 4254 is a peculiar spiral galaxy located at the periphery of the Virgo cluster. It has a prominent $m=1$ spiral pattern, making it look basically one-armed, and its HI distribution and kinematics are quite distorted. Deep VLA HI imaging (Phookun et al. 1993) showed kinematically perturbed, low surface density gas around the galaxy, in particular, an extended very low surface density tail to the northwest of the galaxy. We observed NGC 4254 in HI with the Effelsberg 100-m telescope at 5 different positions: towards the center and at 4 positions located one 9.3' HPBW to the NE, NW, SW, and SE from the center. Our NW spectrum shows $\sim 30 \%$ more low surface density gas than the VLA data, and a bimodal velocity distribution in the part that corresponds to the north-western gas tail.

We investigated if a scenario involving a tidal interaction and subsequent ram pressure stripping could explain these properties. In a first step we modeled a close and rapid encounter between NGC 4254 and another massive galaxy. In this way we were able to reproduce the observed asymmetric spiral structure $\sim 280 \mathrm{Myr}$ after the passage of the perturbing galaxy, for which we identified NGC 4262 as a potential candidate.

In a second step we included the effects of ram pressure stripping that slowly increases with time since we assume that NGC 4254 is entering the Virgo cluster in an almost face-on stripping geometry with an angle of $70^{\circ}$ between the galaxy disk and the orbital plane. At its location at the periphery of the Virgo cluster, the small ram pressure $\left(p_{\text {ram }} \sim 100 \mathrm{~cm}^{-3}\left(\mathrm{~km} \mathrm{~s}^{-1}\right)^{2}\right)$ would not cause significant HI stripping of NGC 4254 without the tidal interaction event that has pulled gas out from the disk. The observed HI properties can only be reproduced if we include the effects of gas expansion and/or evaporation into our simulations. Combining all effects we succeeded in reproducing the main characteristics of the HI spectra, gas distribution, and velocity field, as well as the $m=1$ spiral pattern.

Thus, NGC 4254 has an evolutionary history in the Virgo cluster similar to that of NGC 4654 (Vollmer 2003); both are entering the Virgo cluster for the first time, experienced a tidal interaction and are still undergoing ram pressure stripping.

Acknowledgements. Based on observations with the 100-m telescope of the MPIfR (Max-Planck-Institut für Radioastronomie) at Effelsberg. B.V. would like to thank L. Hernquist for making his program that generates initial conditions available and the NCSA Astronomy Digital Image Library (ADIL) for providing the VLA data cube for this article. This research has made use of the NASA/IPAC Extragalactic Database (NED), which is operated by the Jet Propulsion Laboratory, California Institute of Technology, under contract with the National Aeronautics and Space Administration. We would like to thank the anonymous referee for the useful comments which helped to improve this article.

\section{References}

Cayatte, V., van Gorkom, J. H., Balkowski, C., \& Kotanyi, C. 1990, AJ, 100, 604

Cayatte, V., Kotanyi, C., Balkowski, C., \& van Gorkom, J. H. 1994, AJ, 107, 1003

Combes, F., Dupraz, C., Casoli, F., \& Pagani, L. 1988, A\&A, 203, L9

Cowie, L. L., \& McKee, C. F. 1977, ApJ, 211, 135

de Vaucouleurs, G., de Vaucouleurs, A., Corwin, H. G., et al. 1991, Third Reference Catalogue of Bright Galaxies (New York: Springer) (RC3)

Guharthakurta, P., van Gorkom, J. H., Kotanyi, C. G., \& Balkowski, C. 1988, AJ, 96,851

Gunn, J. E., \& Gott, J. R. 1972, ApJ, 176, 1

Hernquist, L. 1993, ApJS, 86, 389

Iye, M., Okamura, S., Hamabe, M., \& Watanabe, M. 1982, ApJ, 256, 103

Kenney, J. D., \& Young, J. S. 1988, ApJS, 66, 261

Krumm, N., van Driel, W., \& van Woerden, H. 1985, A\&A, 144, 202

Minchin, R., Davies, J., Disney, M., et al. 2005, ApJ, 622, L21

Phookun, B., \& Mundy, L. G. 1995, ApJ, 453, 154

Phookun, B., Vogel, S. N., \& Mundy, L. G. 1993, ApJ, 418, 113

Schindler, S., Binggeli, B., \& Böhringer, H. 1999, A\&A, 343, 420

Sperandio, M., Chincarini, G., Rampazzo, R., \& de Souza, R. 1995, A\&AS, 110, 279

Springel, V., Yoshida, N., \& White, D. M. 2001, NA, 6, 79

van Driel, W., \& van Woerden, H. 1991, A\&A, 243, 71

Vollmer, B. 2003, A\&A, 398, 525

Vollmer, B., \& Huchtmeier, W. 2003, A\&A, 406, 427

Vollmer, B., Cayatte, V., Balkowski, C., \& Duschl, W. J. 2001, ApJ, 561,708

Warmels, R. H. 1988, A\&AS, 72, 427

Wiegel, W. 1994, Diploma Thesis, University of Heidelberg 